\title{
The Clinical Presentation of Subcutaneous Phaeohyphomycosis: A Case Series from Yetebon, Ethiopia
}

\author{
Rachel B. Kang, DPM; Devin C. Simonson, DPM; Sarah E. Stoner, MS;
} Sarah R. Hughes, MD; and William A. Agger, MD

\begin{abstract}
Subcutaneous phaeohyphomycosis is a chronic fungal infection usually found on the lower extremity and feet of agricultural workers in the tropics. It can present with various skin manifestations, verrucous to nodular plaques, and is caused by multiple species of fungi. Laboratory confirmation requires skin samples for pathology and fungal cultures. Cure, often difficult in resource-poor countries, requires months of antifungal therapy. We describe the cases of three men from Ethiopia who were seen and are being treated by American doctors who traveled there on a medical mission.
\end{abstract}

Keywords: Fungal infection; Lower extremity; Subcutaneous phaeohyphomycosis

$\mathrm{S}$ ubcutaneous phaeohyphomycosis is a chronic, indolent infection caused by a multitude of melanized fungal species. Although most fungi produce cell wall melanin, which is considered a pathogenic factor, these infections tend to be caused by fungi that are high melanin producers. ${ }^{1,2}$ Such pigmented fungi are often labeled "dematiaceous"misnomer because this word derives from Greek for "deme," meaning bundle. A specific subset of these subcutaneous fungal infections, characterized by muriform cells (brown pigmented fungal cell collections seen in subcutaneous tissue on microscopy), is called chromoblastomycosis. The latter is commonly associated with the three highly melanized, fungal genera: Fonsecaea, Phialophora, and Cladophialophora. ${ }^{3}$

Collectively, these subcutaneous infections are clinically distinguished from eumycetomas, which also are indolent fungal infections of tropical agricultural laborers; however, eumycetoma infections involve deeper structures, including the bones and joints of the feet and ankles, and are characterized by chronic extrusion of "rice granules" of various colors, including white, dark gray, or even pink or red, composed of fungal colonies. Such eumycetomas are historically labeled as Madura foot, ${ }^{2}$ a deforming disease also caused by chronic bacterial infections, most commonly actinomycetes species.
In the latter, called botryomycosis, the granules extruded are often sulfur colored.

The following, a case series of a subcutaneous phaeohyphomycosis, serves to emphasize the clinical aspects of this indolent infection. These were collected during a short stay by a multidisciplinary medical group who aided a rural medical center located in Yetebon, Ethiopia.

Epidemiology and Clinical Evaluation of the Cases In February 2016, a multidisciplinary medical team from the Midwestern United States traveled to Yetebon, Ethiopia. Yetebon is in a rural, agricultural area located on the Ethiopian plateau along the western edge of Africa's Great Rift Valley. At an altitude of approximately 2500 meters above sea level, Yetebon's climate is semi-arid, it is geologically volcanic with rocky soils, and its natural vegetation in non-cultivated terrain is characterized by mixed grassland and thorny acacia.

While in Yetebon, the visiting medical team, which included podiatry specialists, encountered three patients with similar clinical histories, including daily ambulation across the treacherous terrain of rural Ethiopia while barefoot or wearing only plastic flip flops, an inability to perform regular daily
Corresponding Author: William A. Agger, MD, Gundersen Medical Foundation, 1900 South Avenue, La Crosse, WI 5460I,Tel: (608) 775-6648,

Fax: (608) 775-1565, Email: waagger@gmail.com

\author{
Received: July 10, 2017 \\ Revised: September 6, 2017 \\ Accepted: October 3, 2017
}

doi: $10.3121 / \mathrm{cmr} .2017 .1377$ 


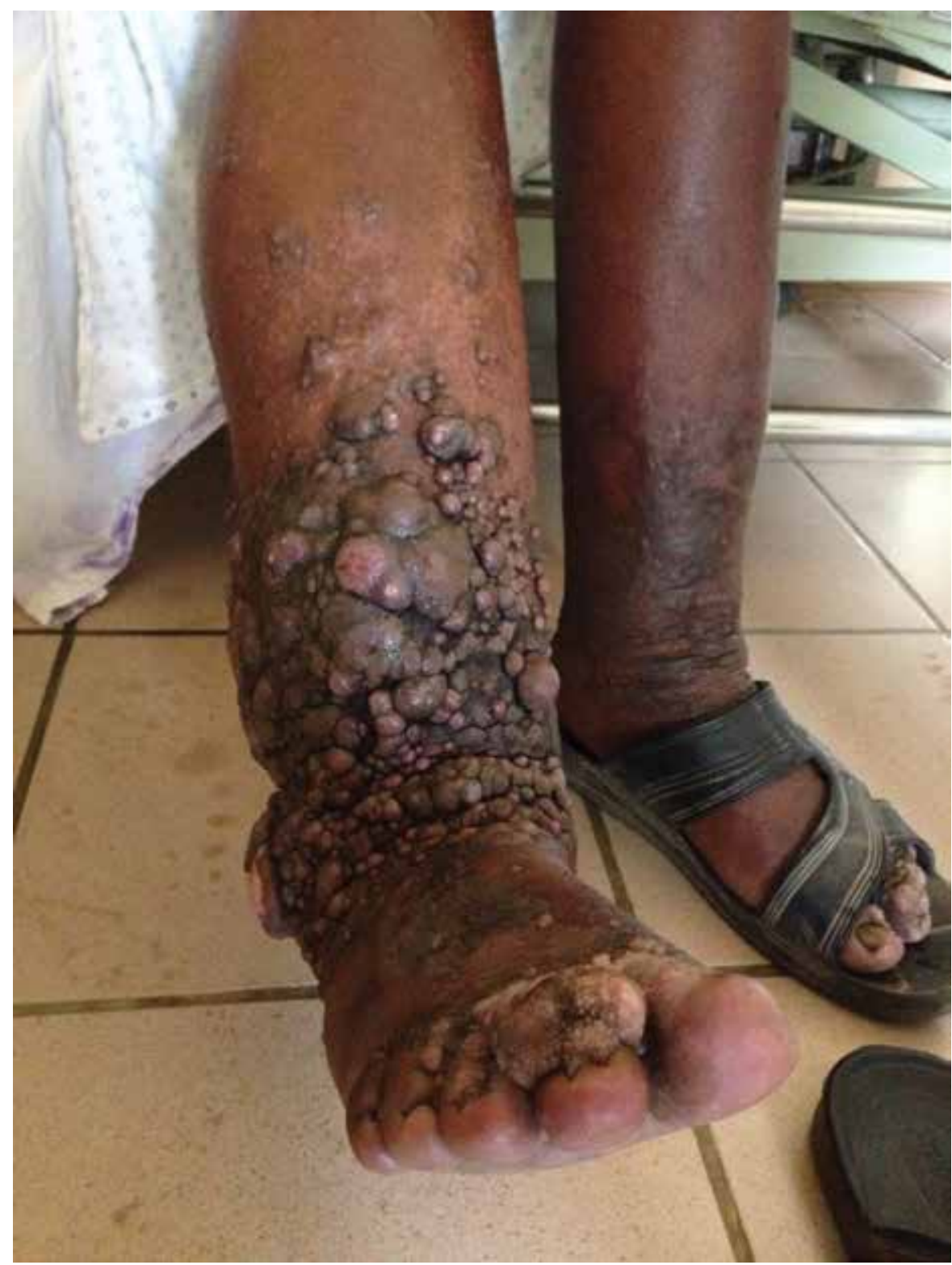

Figure 1. Bilateral lower extremities of a boy, aged 14 years, with an 18-month history of lower extremity edema and skin nodular formation, worse on the right lower extremity.

hygiene due to limited access to clean water, extreme poverty, and a greater than 1-year history of asymmetrical, bilateral lower extremity edema with either a "mossy foot" appearance or multiple edematous nodules located on the foot and/or lower leg. None had the visual "black pepper" spotted lesions commonly seen in classical chromoblastomycosis. The patients were not found to have lower extremity or groin lymph node involvement, which is often found in filarial elephantiasis. None had undergone prior treatment, whether conservative or operative, for their deformities. All three patients had normal, painless lower function of their foot and ankle joints. Therefore, routine radiographs were not performed, nor were magnetic resonance imaging or computed tomography scans available in this rural setting.
Upon receiving the patients' consent, because neither punch biopsy nor local anesthesia were available, epithelial tissue samples were sharply debrided for pathology and fungal cultures.

\section{Case Descriptions}

Patient \#1

A boy, aged 14 years, who lived with his family on a farm, was brought to the clinic on foot from over 20 miles away by his father who was concerned about his son's disfiguring, lower extremity edema. The edema had been present for approximately 18 months and was gradually worsening (Figure 1).

\section{Patient \#2}

A man, aged 65 years, had been experiencing mild lower 


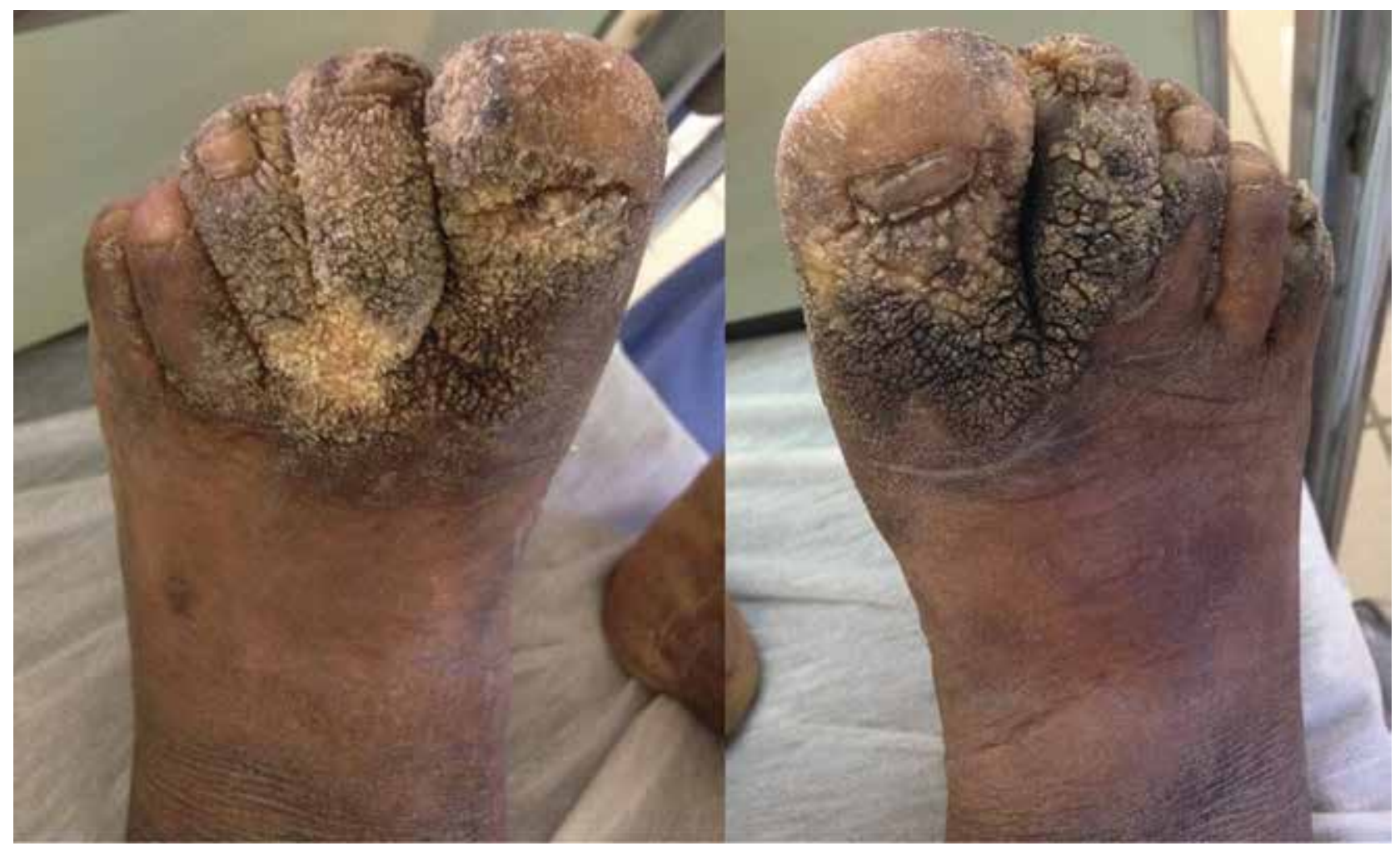

Figure 2. The feet of a man, aged 65 years, with a 4-year history of mild lower extremity edema and verrucous appearance of skin to the toes.

extremity edema and gradually worsening "mossy" appearance on the skin to the toes of bilateral feet, for approximately 4 years (Figure 2). He had not previously sought treatment, but he had heard through some family members that a multidisciplinary medical team was visiting Project Mercy in Yetebon.

\section{Patient \#3}

A man, aged 66 years, who was a farmer living just west of Yetebon in the Gurage Mountains, a range surrounding the rural area of Yetebon, related a 10-year history of edema and nodules to his bilateral lower extremities (Figure 3). He had been seen by an herbal healer who applied many different traditional medical treatments, without improvement.

\section{Fungal Isolation and Identification}

Superficial epidermal samples were obtained from the patients. Tissue microscopy using hematoxylin and eosin (H\&E) stain did not reveal muriform cells (also called copper-penny cells, sclerotic cells, and medlar bodies) typically seen in chromoblastomycosis; however, periodic acid-Schiff (PAS) stain confirmed clusters of yeast forms in all three cases, including rare, melanotic hyphae in patients 2 and 3, and Masson-Fontana stain was weakly positive for melanin in these specimens.

After prolonged specimen transportation, fungal cultures placed on multiple media including inhibitory mold agar (Difco Laboratories, Inc., Detroit, MI) grew Aspergillus niger, Aspergillus (not fumigatus), and Candida parapsilosis complex species from Patient \#2. A melanotic Cladosporium species from Patient \#3 was identified as $C$. pseudocladosporioides based on sequence analysis of the D1/D2 domains of the 28S rRNA gene.

\section{Discussion}

The epidemiology of these three cases from Ethiopia was consistent with that of prior reports of tropical subcutaneous phaeohyphomycosis. ${ }^{1,2}$ Those affected were low-income, agricultural field workers, all men, two of whom were over the age of 30 years. None of them wore protective footwear suitable for the thorn-filled environment where they lived. The infecting fungi, usually considered saprophytes that live in soil or on diverse plants, typically inoculate the patient (ie, host) by accidental injuries from wood splinters and tree thorns. In this situation, even an immunocompetent host can develop smoldering fungal infections. ${ }^{4,5}$ We were able to isolate Cladosporium pseudocladosporioides from Patient \#3. Under laboratory conditions, this species grows poorly at $35^{\circ} \mathrm{C}$, and barely at all at $37^{\circ} \mathrm{C}$. We speculate that this infection occurred in this case due to the inoculation of the distal extremity and the cooler high altitudes of Ethiopia. During microbiologic evaluation, especially from cultures obtained from immunocompetent patients, such saprophytic fungal isolates can be overlooked as laboratory contaminants. ${ }^{6}$

As is usual, the patients included in our report did not recall specific inciting events or injury. Initially, pruritus and 


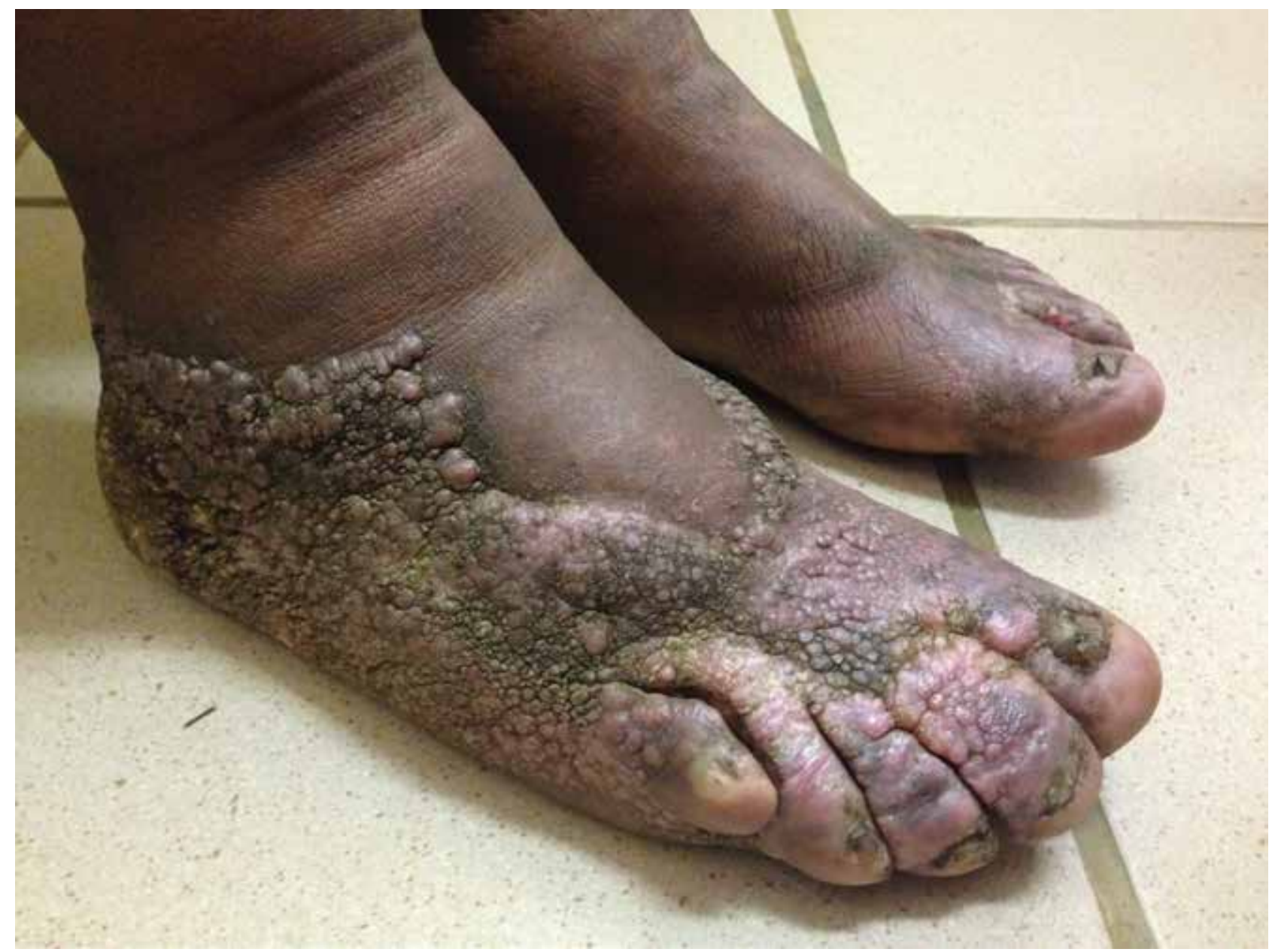

Figure 3. The feet of a man, aged 66 years, with a 10-year history of edema and nodular skin formation to the feet, worse on the right lower extremity.

lymphedema may be the only symptoms. Although the average incubation period from inoculation to disease manifestation varies, it is thought to progress slowly over many years and even decades. Initially, patients may have a solitary, unilateral nodular lesion in the lower extremity, which can gradually increase to a polymorphic, raised, scaly plaque with radial expansion. ${ }^{7}$ In 1950, Carrion ${ }^{8}$ described five clinical forms of chromoblastomycosis (the subset of phaeohyphomycosis with muriform cells), including nodular, tumorous, verrucous, cicatricial, and plaque type. Patients can have more than one type of lesion, especially as disease state progresses, but the most common types, as in these cases, are nodular, tumorous, and verrucous. ${ }^{9}$

Differential considerations for chronic extremity infections include mycobacterial infections, actinomycetoma, and other bacterial botrimycosis; eumycetoma, along with other specific subcutaneous phaeohyphomycosis, such as sporotrichosis; and the classic endemic fungi, such as blastomycosis. ${ }^{2}$

Noninfectious etiologies include squamous cell carcinoma and cutaneous sarcoidosis. Diagnosis can be confirmed with biopsy for pathology and appropriate fungal, mycobacterial, and bacterial cultures. ${ }^{9}$ Suggested initial tissue stains include H\&E and PAS stains, with additional special stains, including acid-fast bacteria (AFB), Grocott's methenamine silver (GMS), and Masson-Fontana (for melanin), depending on the initial results, to aid in the pathologic confirmation of the granulomatous reaction found in many of the chronic infections discussed above. Noteworthy is that these infections can have a paucity of fungal forms in tissues. Therefore, their yeast cells can be difficult to observe, as noted in the most common cause of phaeohyphomycosis in North America, sporotrichosis. Observation of muriform cells is required for the more specific diagnosis of chromoblastomycosis. Cutaneous or subcutaneous biopsy specimens may be obtained after local anesthesia and are ideal for fungal culture, but superficial skin samples can be obtained by minimally invasive technique for fungal culture to support the diagnosis. The diagnosis can be difficult in resource-poor settings, such as that in which these patients were seen. When in doubt, repeat biopsy for cultures and pathology should be done when clinically indicated.

Once diagnosis is made, treatment for cutaneous phaeohyphomycosis can be a therapeutic challenge secondary 
to the recalcitrant nature of the fungal disease, especially for those patients who seek care many years after initial inoculation. Antifungal therapy with itraconazole or other imidizoles, if available and affordable, is recommended over a prolonged period of more than 3 months. ${ }^{2}$ Other antifungal therapies include terbinafine, ${ }^{6}$ saturated solutions of potassium iodine (SSKI), along with either heat or cryotherapy. ${ }^{2}$ Operative resection, while on antifungal therapy, can be successful for localized infections. Unfortunately, relapses are common.

\section{Conclusion}

Subcutaneous phaeohyphomycosis is a chronic, subcutaneous fungal infection initiated by plant matter-associated trauma, usually to the feet and lower legs. Commonly seen in agricultural workers in tropical locations, skin manifestations vary-from verrucous to nodular plaques. Caused by a large variety of melanotic species of fungi, its confirmation requires skin samples for pathology and fungal cultures. Cure, often difficult in resource-poor countries, requires months of antifungal therapy often coupled with cryotherapy or surgery. In our cases, therapy has been challenged, owing to the distant and rural location of the cases in a developing nation fraught with political disruptions, but efforts are ongoing.

\section{References}

1. Revankar SG, Patterson JE, Sutton DA, Pullen R, Rinaldi MG. Disseminated phaeohyphomycosis: review of an emerging mycosis. Clin Infect Dis 2002;34(4):467-476.

2. Revankar SG, Sutton DA. Melanized fungi in human disease. Clin Microbiol Rev 2010;23(4):884-928.

3. Esterre P. Chromoblastomycosis. In: Merz WG, Hay RJ, eds. Medical mycology, vol. 5. Topley and Wilson's microbiology and microbial infections. 10th ed. London: Hodder Arnold; 2005:356-366.

4. Revankar SG. Phaeohyphomycosis. Infect Dis Clin North Am 2006;20(3):609-620.

5. Rinaldi MG. Phaeohyphomycosis. Dermatol Clin 1996; 14(1):147-153.

6. Agger WA, Andes D, Burgess JW. Exophiala jeanselmei infection in a heart transplant recipient successfully treated with oral terbinafine. Clin Infect Dis 2004;38(11):e112-e115.

7. Esterre P, Andriantsimahavandy A, Ramarcel ER, Pecarrere JL. Forty years of chromoblastomycosis in Madagascar: a review. Am J Trop Med Hyg 1996;55(1):45-47.

8. Carrion AL. Chromoblastomycosis. Ann N Y Acad Sci 1950; 50(10):1255-1282.

9. Queiroz-Telles F, McGinnis MR, Salkin I, Graybill JR. Subcutaneous mycoses. Infect Dis Clin North Am 2003; 17(1):59-85, viii.

\section{Author Affiliations}

Rachel B. Kang, DPM*; Devin C. Simonson, DPM广; Sarah E. Stoner, MS ; Sarah R. Hughes, MD§; and William A. Agger, $M D^{*}, \boldsymbol{\uparrow}$

*Department of Medical Education, Gundersen Medical Foundation, La Crosse, Wisconsin, USA †Department of Podiatry, Gundersen Health System; La Crosse, Wisconsin, USA

‡Department of Microbiology, Gundersen Health System, La Crosse, Wisconsin, USA

§Department of Pathology, Gundersen Health System, La

Crosse, Wisconsin, USA

ๆInfectious Disease Section, Gundersen Health System, La

Crosse, Wisconsin, USA

Note in Proof

After acceptance, the corresponding author requested the inclusion of Sarah R. Hughes, MD to the author list indicating her name was erroneously omitted from the original submission. An updated copyright transfer agreement including Dr. Hughes' contributions and signature, and a letter signed by all original authors agreeing to the addition of Dr. Hughes to the paper were provided as documentation. 